\title{
Content Based Image Retrieval Using Color Histogram
}

\author{
Nitin Jain \\ Assistant Professor, Lokmanya Tilak College of Engineering, Navi Mumbai, India. \\ Dr. S. S. Salankar \\ Professor, G.H. Raisoni College of Engineering, Nagpur, India.
}

\begin{abstract}
In this paper, we present content based image retrieval using color Histogram. Color algorithm using color histogram was applied. Humans tend to differentiate images based on color, therefore color features are mostly used in CBIR. Color histogram is mostly used to represent color features but it cannot entirely characterize the image. The results clearly indicate that Color Histogram techniques give better precision and recall.
\end{abstract}

Keywords - Content Based Image Retrieval, Color Histogram, Precision, Recall.

\section{INTRODUCTION}

In this era of information technology, all areas of human life including commerce, government, academics, hospitals, crime prevention, surveillance, engineering, architecture, journalism, fashion and graphic design, and historical research use images for efficient services. A large collection of images is referred to as image database. An image database is a system where image data are integrated and stored [1]. Image data include the raw images and information extracted from images by automated or computer assisted image analysis. The police maintain image database of criminals, crime scenes, and stolen items. In the medical profession, X-rays and scanned image database are kept for diagnosis, monitoring, and research purposes. In architectural and engineering design, image database exists for design projects, finished projects, and machine parts. In publishing and advertising, journalists create image databases for various events and activities such as sports, buildings, personalities, national and international events, and product advertisements. In historical research, image databases are created for archives in areas [1]. The CBIR technique uses image content to search and retrieve digital images stored in large database. Content based image retrieval is a set of techniques for retrieving semantically-relevant images from an image database based on automatically-derived image features [2] [3] [4]. The main goal of CBIR is efficiency during image indexing and retrieval, thereby reducing the need for human intervention in the indexing process. The computer must be able to retrieve images from a database without any human interruption on specific domain such color and texture. One of the main tasks for CBIR systems is similarity comparison; extracting feature of every image based on its pixel values and devising rules for comparing images. These features become the image representation for measuring similarity with other images in the database. An image is compared to other images by calculating the difference between their corresponding features. Some of the existing CBIR systems extract features from the entire image instead of certain regions in it. These features are referred to as Global features. Histogram search algorithms [3] characterize an image by its color distribution or histogram. The drawback of a global histogram representation is that information about object location, shape and texture is discarded. Color histogram search is sensitive to intensity variations and color distortions. The color layout approach attempts to overcome the drawback of histogram search. In simple color layout indexing [3], images are partitioned into blocks and the average color of each block is stored. Thus, the color layout is essentially a low resolution representation of the original image. In the field of computer vision and image processing, there is no clear-cut definition of texture. This is because available texture definitions are based on texture analysis methods and the features extracted from the image. Texture properties are the visual patterns in an image that have properties of homogeneity that do not result from the presence of only a single color or intensity. The different texture properties as perceived by the human eye are, for example, regularity, directionality, smoothness, and coarseness, see Fig. 1. 


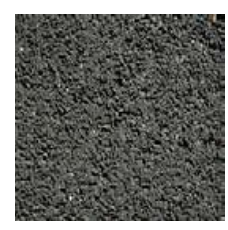

Coarse

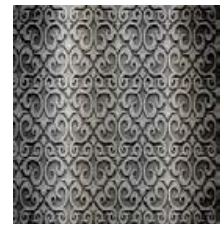

Regular

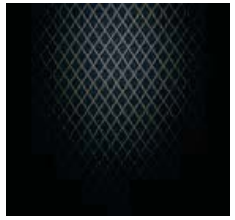

Directional

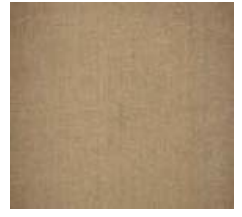

Fine

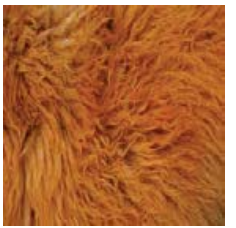

Irregular

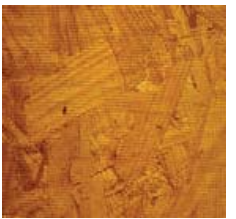

Un-directional
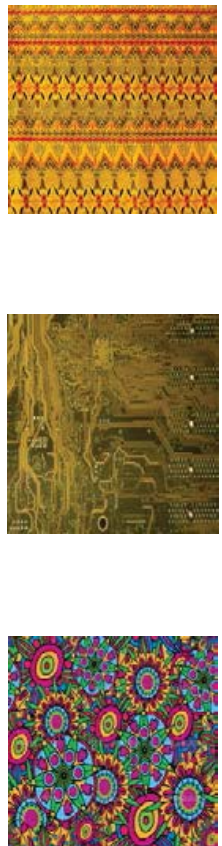

(b) Complex

(a) Simple

Fig. 1. Images of Simple \& Complex Texture

Since there is no accepted mathematical definition for texture, many different methods for computing texture features have been proposed over the years. Unfortunately, there is still no single method that works best with all types of textures. According to [5], the commonly used methods for texture feature description are statistical, model-based, and transform-based methods. The word transform refers to a mathematical representation of an image. There are several texture classifications using transform domain features in the past, such as discrete Fourier transform, discrete wavelet transforms, and Gabor wavelets. Gabor filter has been shown to be very efficient [5] and have also shown that image retrieval using Gabor features outperforms that using other transform features. In this paper, we present two features color and texture extraction algorithms. Color histogram is mostly used to represent color features but it cannot entirely characterize the image. Color Histogram is also rotation invariant about the view axis. Gabor filter, a tool for texture feature extraction has proved to be very effective in describing visual content via multi-resolution analysis. Texture feature extraction based on Gabor filter is presented.

\section{COLOR HISTOGRAM}

Color is a powerful descriptor that simplifies object identification, and is one of the most frequently used visual features for content-based image retrieval. To extract the color features from the content of an image, a proper color space and an effective color descriptor have to be determined. The purpose of a color space is to facilitate the specification of colors. Each color in the color space is a single point represented in a coordinate system. Several color spaces, such as RGB, HSV, CIE L*a*b, and CIE L*u*v, have been developed for different purposes [6]. Although there is no agreement on which color space is the best for CBIR, an appropriate color system is required to ensure perceptual uniformity. Therefore, the RGB color space, a widely used system for representing color images, is not suitable for CBIR because it is a perceptually non-uniform and devicedependent system [7]. The most commonly used method to represent color feature of an image is the color histogram. A color histogram is a type of bar graph, where the height of each bar represents an amount of particular color of the color space being used in the image [6]. The bars in a color histogram are named as bins and they represent the $\mathrm{x}$-axis. The number of bins depends on the number of colors there are in an image. The number of pixels in each bin denotes y-axis, which shows how many pixels in an image are of a particular color. The color histogram can not only easily characterize the global and regional distribution of colors in an image, but also be invariant to rotation about the view axis. In color histograms, quantization is a process where number of bins is reduced by taking colors that are similar to each other and placing them in the same bin. Quantizing reduces the space required to store the histogram information and time to compare the histograms. Obviously, 
quantization reduces the information regarding the content of images; this is the tradeoff between space, processing time, and accuracy in results [8]. Color histograms are classified into two types, global color histogram $(\mathrm{GCH})$ and local color histogram $(\mathrm{LCH})$. A GCH takes color histogram of whole image and thus represents information regarding the whole image, without concerning color distribution of regions in the image. In the contrary, an LCH divides an image into fixed blocks or regions, and takes the color histogram of each of those blocks. LCH contains more information about an image, but when comparing images, it is computationally expensive. GCH is known as a traditional method for retrieving color based images. Since it does not include color distribution of the regions, when two GCHs are compared, one might not always get a proper result when viewed in terms of similarity of images [9]. Fig. 2 shows the image with its color histogram.
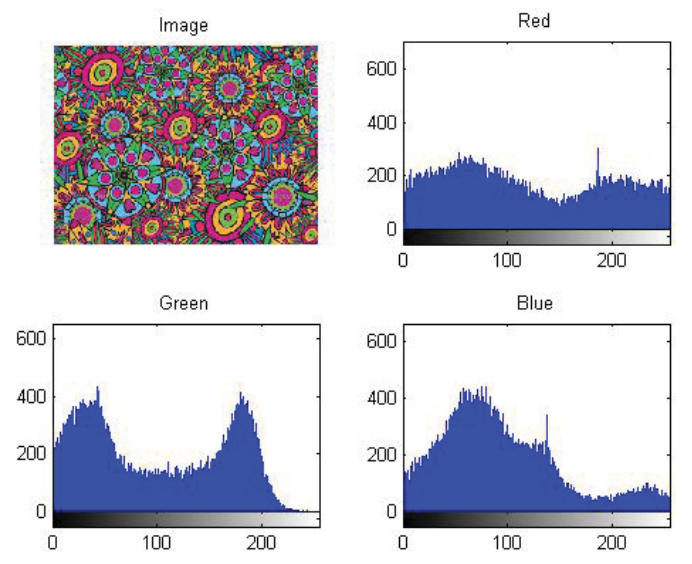

Fig. 2. Image and its corresponding color Histogram

\section{PERFORMANCE EVALUATION}

The performance of retrieval system is measured using the standard procedure in terms of the precision and recall values [10]. Recall measures the ability of the system to retrieve all the models that are relevant, while precision measures the ability of the system to retrieve only the models that are relevant. Precision, $\mathrm{P}$, is defined as the ratio of the number of retrieved relevant images to the total number of retrieved images. Let the number of all retrieved images be $\mathrm{n}$, and let $\mathrm{r}$ be the number of relevant images according to the query then the precision value is: $\mathrm{P}=\mathrm{r} / \mathrm{n}$. Precision $\mathrm{P}$ measures the accuracy of the retrieval. Recall $\mathrm{R}$ is defined as the ratio of relevant images retrieved to the total number of relevant images. Let $\mathrm{m}$ be the number of relevant images retrieved according to query then recall value is $\mathrm{R}=\mathrm{m} / \mathrm{r}$.

\section{RESULTS \& DISCUSSION}

The proposed method is applied on a general-purpose set of containing 500 images of the database, in JPEG format of size $300 \times 350$. These images are grouped into five different categories with each containing 100 images. The images in the same category are considered as similar images. The five different categories are: (i) brain, (ii) retina, (iii) coins, (iv) sun and (v) leaves. The objective of the paper is to design a CBIR system that is simple to use, easy to handle large Image data bases, and fastest to retrieve images using low-level features such as color and texture. The similarity between two images (represented by their feature values) is defined by a similarity measure. Selection of similarity metrics has a direct impact on the performance of content-based image retrieval. The kind of feature vectors selected determines the kind of measurement that will be used to compare their similarity [4]. If the features extracted from the images are presented as multi-dimensional points, the distances between corresponding multi-dimensional points can be calculated. Fig 3 shows the snapshot of the retrieved images of coins. Table 1 shows the precision and recall for the technique described in this paper. The experiments were carried on Intel core i5, $2.4 \mathrm{GHz}$ processor with 4GB RAM. 

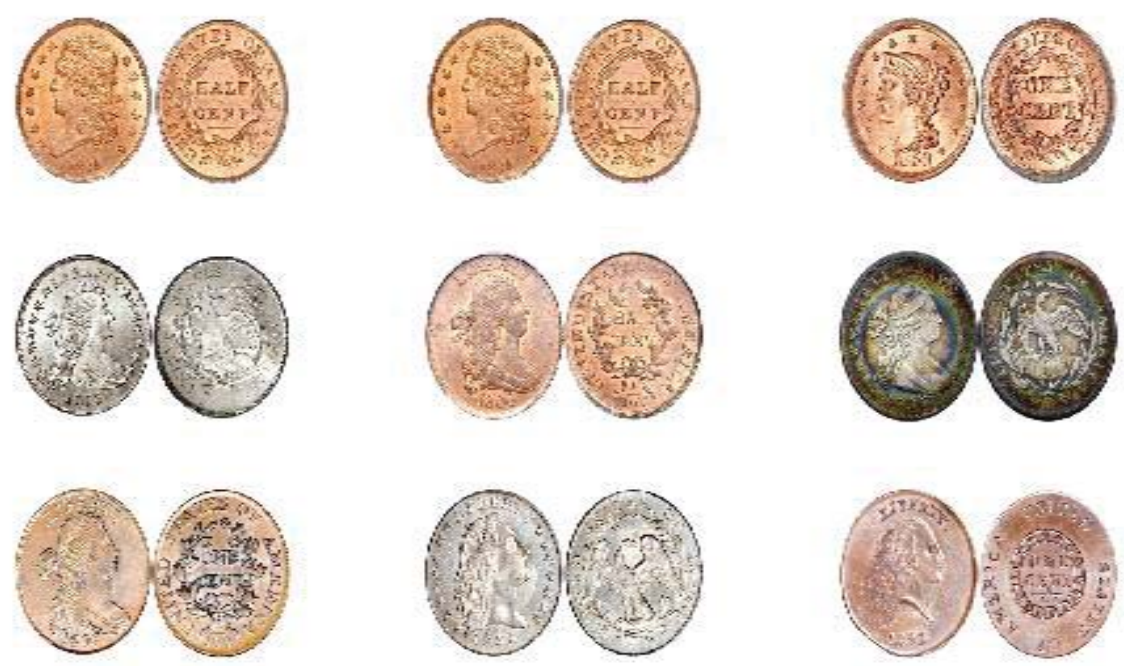

Fig. 3 Snapshot of Retrieved Images of Coins

Table 1 Precision and Recall

\begin{tabular}{|c|c|c|}
\hline Image Categories & Precision & Recall \\
\hline Brain & 0.38 & 0.47 \\
\hline Retina & 0.24 & 0.56 \\
\hline Coins & 0.6 & 0.49 \\
\hline Sun & 0.3 & 0.45 \\
\hline Leaves & 0.24 & 0.4 \\
\hline Average & 0.352 & 0.474 \\
\hline
\end{tabular}

\section{CONCLUSION}

In this paper, we presented CBIR system using two feature color and texture extraction algorithms. Color and Texture features extraction algorithm using color histogram and Gabor Wavelet Transform respectively were applied. Color histogram is mostly used to represent color features but it cannot entirely characterize the image. Gabor filter, a tool for texture feature extraction has proved to be very effective in describing visual content via multi-resolution analysis. The results clearly indicate that combination of Gabor Wavelet Transform and Color Histogram techniques improves precision and recall.

\section{REFERENCES}

[1] Chi Kuo Chang, "Image Information Systems," Proc. of IEEE Pattern Recognition, vol. 73, no 4, pp.754 - 766, April 1985.

[2] S. Gerard, C. Buckely, "Term-Weighting Approaches in Automatic Text Retrieval," Information Processing and Management, vol. 24, no.5, pp. 513-523, Jan. 1988.

[3] Y. Chen, J. Wang, "Image Categorization by Learning and Reasoning with Regions," Journal of Machine Learning Research, vol. 5, pp. 913-939, May 2004.

[4] F. Long, H. Zhang, H. Dagan, and D. Feng, "Fundamentals of content based image retrieval," in D. Feng, W. Siu, H. Zhang (Eds.): "Multimedia Information Retrieval and Management. Technological Fundamentals and Applications," Multimedia Signal Processing Book, Chapter 1, Springer-Verlag, Berlin Heidelberg New York, 2003, pp.1-26.

[5] B. Manjunath and W. Ma, "Texture features for Browsing and retrieval of image data," IEEE transactions on pattern analysis and machine intelligence, vol. 18. No. 8, pp. 837-842, August 1996 
[6] R. Gonzales, R. E. Woods, "Digital Image Processing,” 2nd Ed., New Jersey Prentice Hall, 2002.

[7] T. Gevers and A. Smeulders, "Pictoseek: Combining color and shape invariant features for image retrieval," IEEE Trans. Image Processing, vol. 9, no. 1, pp.102-119, Nov. 2000

[8] M. Lew, N. Sebe, C. Djeraba and R. Jain, "Content-Based Multimedia Information Retrieval: State of the Art and Challenges," ACM Transactions on Multimedia Computing, Communications and Applications, vol. 2, no. 1, pp. 1-19, February 2006.

[9] J. Fuertes, M. Lucena, N. Perez, and J. Martinez, "A Scheme of Color Image Retrieval from Databases," Pattern Recognition Letters, vol. 22, pp.323-337, June 2001.

[10] Banerjee, M., Kundu, M.K., Maji, P.: ‘Content-based image retrieval using visually significant point features', Fuzzy Sets Syst., 160, pp. 3323-334, 2009. 